\title{
The particle and magnetic environments surrounding close-in exoplanets
}

\author{
A. A. Vidotto ${ }^{1}$, R. Fares ${ }^{2}$, M. Jardine ${ }^{3}$, C. Moutou ${ }^{4,5}$ and \\ J.-F. Donati ${ }^{6,7}$ \\ ${ }^{1}$ Université de Genève, Chemin des Maillettes 51, Versoix, CH-1290, Switzerland \\ email: Aline.Vidotto@unige.ch \\ ${ }^{2}$ INAF, Osservatorio Astrofisico di Catania, Via Santa Sofia, 78, 95123 Catania, Italy \\ ${ }^{3}$ SUPA, University of St Andrews, North Haugh, St Andrews, KY16 9SS, UK \\ ${ }^{4}$ CFHT, CNRS, 65-1238 Mamalahoa Hwy, Kamuela HI 96743, USA \\ ${ }^{5}$ Aix Marseille Université, CNRS, LAM UMR 7326, 13388, Marseille, France \\ ${ }^{6}$ Université de Toulouse, UPS-OMP, IRAP, 14 avenue E. Belin, Toulouse, F-31400, France \\ ${ }^{7}$ CNRS, IRAP / UMR 5277, Toulouse, 14 avenue E. Belin, F-31400, France
}

\begin{abstract}
The proper characterisation of stellar winds is essential for the study of propagation of eruptive events (flares, coronal mass ejections) and the study of space weather events on exoplanets. Here, we quantitatively investigate the nature of the stellar winds surrounding the hot Jupiters HD46375b, HD73256b, HD102195b, HD130322b, HD179949b. We simulate the three-dimensional winds of their host stars, in which we directly incorporate their observed surface magnetic fields. With that, we derive the wind properties at the position of the hotJupiters' orbits (temperature, velocity, magnetic field intensity and pressure). We show that the exoplanets studied here are immersed in a local stellar wind that is much denser than the local conditions encountered around the solar system planets (e.g., 5 orders of magnitude denser than the conditions experienced by the Earth). The environment surrounding these exoplanets also differs in terms of dynamics (slower stellar winds, but higher Keplerian velocities) and ambient magnetic fields ( 2 to 3 orders of magnitude larger than the interplanetary medium surrounding the Earth). The characterisation of the host star's wind is also crucial for the study of how the wind interacts with exoplanets. For example, we compute the exoplanetary radio emission that is released in the wind-exoplanet interaction. For the hot-Jupiters studied here, we find radio fluxes ranging from 0.02 to $0.13 \mathrm{mJy}$. These fluxes could become orders of magnitude higher when stellar eruptions impact exoplanets, increasing the potential of detecting exoplanetary radio emission.
\end{abstract}

Keywords. stars: low-mass, brown dwarfs, stars: magnetic fields, stars: winds, outflows, interplanetary medium, planets and satellites: general

\section{Introduction}

The interplanetary medium that surrounds exoplanets is filled by the quiescent stellar wind particles and the embedded stellar magnetic field. Eruptive events, such as flares and coronal mass ejections, also permeate the interplanetary medium, but these events, especially for stars that are not very active, are often of short duration and, after their passage, the interplanetary medium recovers to its quiescent state. Characterising the stellar wind is essential for the study of propagation of eruptive events (e.g., Loesch et al. 2011). In addition, a better knowledge of stellar winds is also crucial for the study of how the wind interacts with exoplanets and can supply more detailed diagnostics of its interaction with exoplanets (Vidotto et al. 2011a,b, 2012, 2014a,b, 2015; Llama et al. 2013). 
Here, we summarise the results of our modelling efforts of the winds of five planethosting stars of spectral types F8 to K1, namely: HD 46375, HD 73256, HD 102195, HD 130322 and HD 179949. A full description of this work is found in Vidotto et al. (2015).

\section{Stellar wind characterisation}

Stellar winds are three-dimensional (3D) in nature, where complex interactions of a rotating, magnetised plasma take place. We use the 3D magnetohydrodynamics (MHD) numerical code BATS-R-US (Powell et al. 1999; Tóth et al. 2012) to simulate the stellar winds. The radial component of the magnetic field $B_{r}$ anchored at the base of the wind, is reconstructed from observations (Fares et al. 2012, 2013), from a series of circular polarisation spectra using the Zeeman-Doppler Imaging (ZDI) technique (e.g., Donati \& Brown 1997; Donati et al. 2006). At the base of the wind $\left(r=R_{\star}\right)$, we adopt a wind temperature $T_{0}=2 \times 10^{6} \mathrm{~K}$ and number density $n_{0}=10^{9} \mathrm{~cm}^{-3}$ for all the stars in our sample. At the initial state of the simulations, we assume that the wind is thermally driven (Parker 1958), with a polytropic index of $\gamma=1.1$, similar to the effective adiabatic index measured in the solar wind (Van Doorsselaere et al. 2011). As the simulations evolve in time, both the wind particles and magnetic field lines are allowed to interact with each other. The relaxed solution, obtained self-consistently, is found when the system reaches steady state in the reference frame corotating with the star.

In our simulations, we find that the stellar mass-loss rates $\dot{M}$ range from $\sim 2$ to $8 \times 10^{-13} \mathrm{M}_{\odot} \mathrm{yr}^{-1}$. The top left panel of Figure 1 shows the final configuration of the stellar magnetic field lines derived for one of our studied targets (HD179949). The top right panel shows the Alfvén surface derived in our calculations - beyond this surface, the stellar wind is super-Alfvénic. The Alfvén surface is crucial for deriving the amount of angular momentum that stellar winds carry along (e.g., Vidotto et al. 2014b).

All the stars in our sample host giant planets orbiting at close distances, with semimajor axes of about 9 to 23 stellar radii (i.e., about 9 to 4 times closer than the orbit of Mercury). The orbital planes of the planets considered in this work are not known. Here, we assume their orbits lie in the equatorial plane of the star. The orbital radius (in blue) of the hot-Jupiter HD179949b is shown for comparison in the top right panel of Figure 1 Note that this planet orbits in the super-Alfvénic regime.

Table 1 shows the derived characteristics of the local environment surrounding the hotJupiters in our sample. We note that all the quantities we derived have some variability as the planet moves along its orbit. This is because the stellar wind is inhomogeneous, so that the planets interact with the varying environment of the star along their orbits. The wind velocities we found at the position of these hot Jupiters are lower than the wind velocity at around the Earth (compare values from Table 1 and the typical solar wind velocities $\sim 400$ to $800 \mathrm{~km} \mathrm{~s}^{-1}$, depending on the activity phase of the Sun). The densities around these planets are typically 5 orders of magnitude larger than the density around the Earth of a few particles per cubic centimetre. The values of the interplanetary magnetic field at the orbits of the hot-Jupiters are more than 100 times larger than the interplanetary magnetic field at the Earth's orbit (compare 0.01 to $0.05 \mathrm{mG}$ to the values presented in Table 1). The values of the local total pressure (sum of thermal, magnetic and ram pressure) are within $(0.58-4.1) \times 10^{-4} \mathrm{dyn}_{\mathrm{cm}}^{-2}$, which are about 4 orders of magnitude larger than the ram pressure of the solar wind at the Earth's orbit $\left(1.8 \times 10^{-8} \mathrm{dyn} \mathrm{cm}^{-2}\right.$, See et al. 2014$)$.

The bottom panel of Figure 1 shows the total pressure of the stellar wind in the equatorial plane of the star HD179949. The orbital radius is depicted by the white circle, 


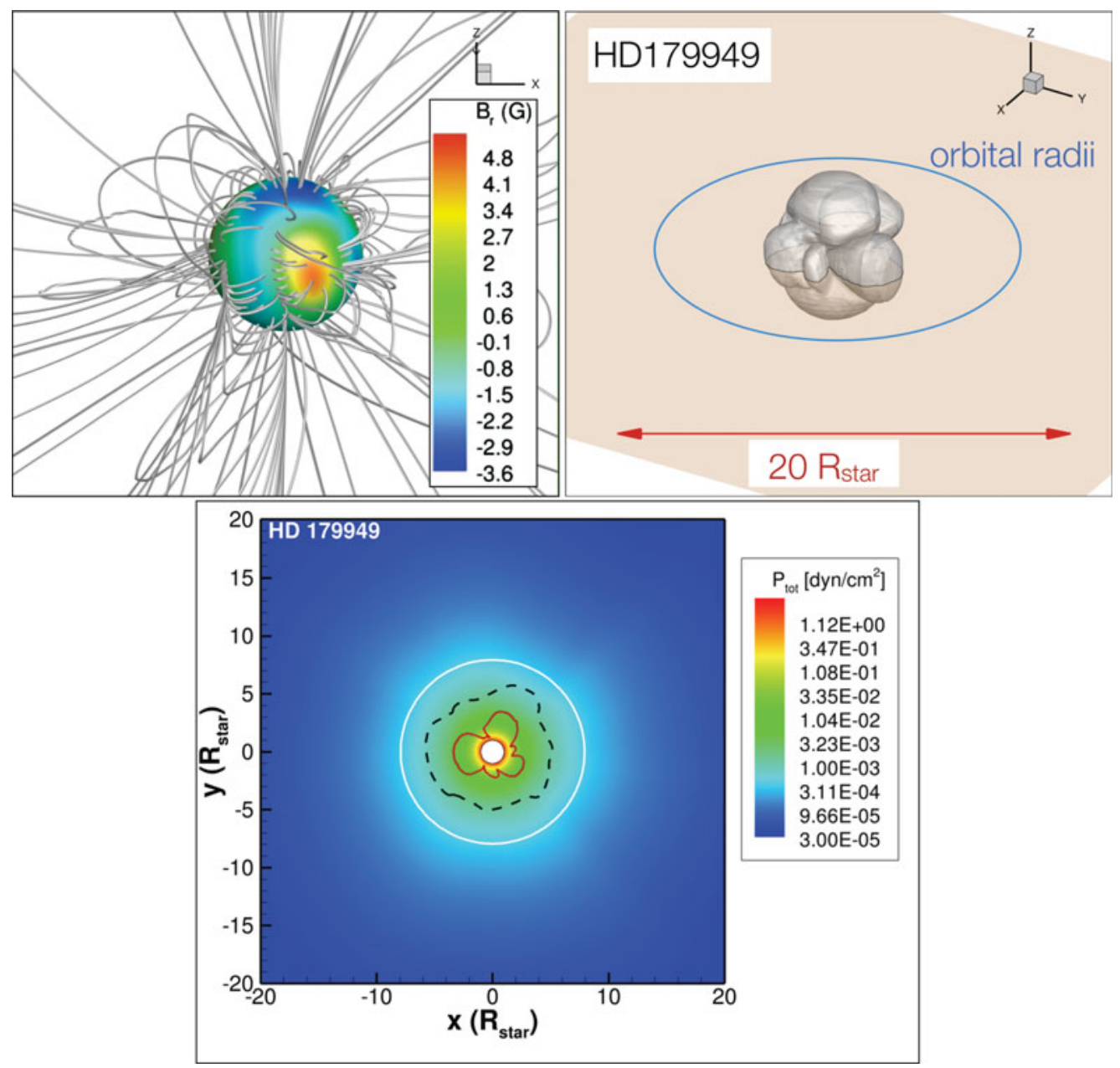

Figure 1. Top left: the final configuration of the stellar magnetic field lines derived for HD179949. The observationally reconstructed surface stellar magnetic field (Fares et al. 2012) is shown in color. Top right: the Alfvén surface derived in our calculations. Also shown for comparison is the orbital radius of the hot-Jupiter HD179949b, which orbits in the super-Alfvénic regime. Bottom: total pressure of the stellar wind (sum of thermal, magnetic and ram pressure) in the equatorial plane of the star. The orbital radius is depicted by the white circle, the Alfvén surface by the red iso-contour, and the magnetosonic surface by the black. After Vidotto et al. (2015).

the Alfvén surface by the red iso-contour, and the magnetosonic surface by the black iso-contour. We show that HD179949b orbit is super-magnetosonic, indicating that a bow shock is formed surrounding this planet. Similar results are found for the remaining systems, but are not shown here due to space limitation. We point the interested reader towards the original work (Vidotto et al. 2015) for further details.

\section{Planetary radio emission}

The stellar wind that impacts on the planet produces energetic particles that are captured by the planet's magnetic field, emitting cyclotron radiation at radio wavelengths. This emission depends on the planet's magnetic field intensity and on the stellar wind 
Table 1. Derived characteristics of local environment surrounding the hot-Jupiters in our sample. The columns are, respectively, the planet name, the averages of the local velocity of the wind in the reference frame of the planet $\langle u\rangle$, wind density $\langle n\rangle$, magnetic field strength $\langle|B|\rangle$, wind temperature $\langle T\rangle$, total pressure $\left\langle p_{\text {tot }}\right\rangle$ (sum of the thermal, magnetic and ram pressures). These quantities were averaged over the subplanetary longitude. Values in brackets represent the minimum and maximum values of the averaged quantity.

\begin{tabular}{|c|c|c|c|c|c|}
\hline $\begin{array}{l}\text { Planet } \\
\text { ID }\end{array}$ & $\begin{array}{c}\langle u\rangle \\
\left(\mathrm{km} \mathrm{s}^{-1}\right)\end{array}$ & $\begin{array}{c}\langle n\rangle \\
\left(10^{5} \mathrm{~cm}^{-3}\right)\end{array}$ & $\begin{array}{l}\langle|B|\rangle \\
(\mathrm{mG})\end{array}$ & $\begin{array}{c}\langle T\rangle \\
\left(10^{6} \mathrm{~K}\right)\end{array}$ & $\begin{array}{c}\left\langle p_{\text {tot }}\right\rangle \\
\left(10^{-4} \frac{\mathrm{dyn}}{\mathrm{cm}^{2}}\right)\end{array}$ \\
\hline HD 46375b & $\begin{array}{c}234 \\
{[228,242]}\end{array}$ & $\begin{array}{c}1.8 \\
{[1.7,2.0]}\end{array}$ & $\begin{array}{c}8.8 \\
{[0.55,11]}\end{array}$ & $\begin{array}{c}0.87 \\
{[0.86,0.91]}\end{array}$ & $\begin{array}{c}1.1 \\
{[1.0,1.2]}\end{array}$ \\
\hline HD 73256b & $\begin{array}{c}263 \\
{[217,345]}\end{array}$ & $\begin{array}{c}2.0 \\
{[1.6,2.6]}\end{array}$ & $\begin{array}{c}17 \\
{[2.6,26]}\end{array}$ & $\begin{array}{c}1.1 \\
{[0.91,1.6]}\end{array}$ & $\begin{array}{c}1.6 \\
{[1.0,2.7]}\end{array}$ \\
\hline HD $102195 b$ & $\begin{array}{c}288 \\
{[240,338]}\end{array}$ & $\begin{array}{c}1.5 \\
{[1.1,2.0]}\end{array}$ & $\begin{array}{c}14 \\
{[3.6,18]}\end{array}$ & $\begin{array}{c}0.96 \\
{[0.87,1.2]}\end{array}$ & $\begin{array}{c}1.3 \\
{[1.1,1.6]}\end{array}$ \\
\hline HD $130322 b$ & $\begin{array}{c}322 \\
{[316,334]}\end{array}$ & {$\left[\begin{array}{c}0.6 \\
{[0.6,0.7}\end{array}\right]$} & $\begin{array}{c}2.3 \\
{[0.36,2.9]}\end{array}$ & $\begin{array}{c}0.78 \\
{[0.77,0.79]}\end{array}$ & $\begin{array}{c}0.62 \\
{[0.58,0.69]}\end{array}$ \\
\hline HD $179949 b$ & $\begin{array}{c}243 \\
{[225,257]}\end{array}$ & $\begin{array}{c}5.9 \\
{[5.5,6.2]}\end{array}$ & $\begin{array}{c}9.6 \\
{[1.4,15]}\end{array}$ & $\begin{array}{c}0.97 \\
{[0.96,0.99]}\end{array}$ & $\begin{array}{c}3.8 \\
{[3.1,4.1]}\end{array}$ \\
\hline
\end{tabular}

power: it implies that the stronger is the stellar wind, the more luminous is the planet. As such radio emission is observed in the Solar System (Zarka 2007), there are expectations that close-in exoplanets will exhibit comparable radiation. In particular, hot-Jupiters are expected to be much more luminous than the most luminous planet in our solar System, Jupiter (e.g., Farrell et al. 1999; Grießmeier et al. 2005; Zarka 2007; Jardine \& Cameron 2008; Vidotto et al. 2010; See et al. 2015). This is because hot-Jupiters are located much closer to their stars, interacting with portions of the host-star's wind that has larger kinetic and magnetic energies available to power planetary radio emission. So far, radio signatures of close-in exoplanets have not yet been detected (e.g. Bastian et al. 2000; Lazio et al. 2004; Smith et al. 2009; Hallinan et al. 2013) and one possible reason for that may be due to the lack of instrumental sensitivity in the appropriate frequency range of the observations (Bastian et al. 2000). This picture, however, might be changing, as possible hints of exoplanetary radio emission have recently been reported (Lecavelier des Etangs et al. 2013; Sirothia et al. 2014).

We use the results of our stellar wind simulations to calculate the kinetic power of the wind, at the orbital radii of the hot-Jupiters studied here. Our approach follows closely the one in Vidotto et al. (2012). Here, we adopt a planetary magnetic field intensity which is similar to the value of Jupiter. We find that the predicted emission frequency occurs at $\sim 36 \mathrm{MHz}$ and the radio fluxes range between 0.02 and $0.13 \mathrm{mJy}$ among all the cases studied here. Values of radio fluxes such as these should be challenging to be observed with present-day technology, such as with LOFAR, whose sensitivity at 20 to $40 \mathrm{MHz}$ is $\gtrsim 30$ to $3 \mathrm{mJy}$, respectively, for a one-hour integration time (Grießmeier et al. 2011). It is likely, however, that even these small radio fluxes will be detectable with future higher sensitivity arrays, such as the SKA-low array system.

It is also worth comparing the emission calculated here and the values calculated for $\tau$ Boo b and HD 189733b. Using the same radio emission model presented here, Vidotto et al. (2012) estimated the radio flux of $\tau$ Boo b at different epochs of the host star's magnetic cycle. They found the radio flux of $\tau$ Boo b to be of the order of $0.5-0.9$ mJy. The radio flux of HD $189733 \mathrm{~b}$ was estimated to be on average $0.2-0.5 \mathrm{mJy}$ with a peak at $0.98 \mathrm{mJy}$ (Vidotto et al. 2015). The radio fluxes computed for $\tau$ Boo b and HD 189733b are therefore considerably larger than the values computed for the exoplanets presented here, having better prospects for being detected. 


\section{Summary}

The hot-Jupiters in our sample interact with much denser winds that have larger densities and ram pressures than those typically found around the planets in the solar System. In addition, because the hot-Jupiters are located much closer to the star, the stellar magnetic field at the orbit of these planets has also a larger strength compared to the interplanetary magnetic field strength of our solar System planets. Because of these extreme environments, the interactions between close-in planets and the stellar wind are believed to be stronger than those found in the solar system. Here, we computed the exoplanetary radio emission that is released in the wind-exoplanet interaction. For the hot-Jupiters studied here, we find radio fluxes ranging from 0.02 to $0.13 \mathrm{mJy}$. These fluxes could become orders of magnitude higher when stellar eruptions impact exoplanets, increasing the potential of detecting exoplanetary radio emission.

\section{Acknowledgements}

AAV acknowledges support from the Swiss National Science Foundation through an Ambizione Fellowship. The results of this work are based on observations acquired at CFHT/ESPaDOnS and TBL/NARVAL. This work was carried out using the BATS-RUS tools developed at The University of Michigan Center for Space Environment Modeling (CSEM) and made available through the NASA Community Coordinated Modeling Center (CCMC). This work was supported by a grant from the Swiss National Supercomputing Centre (CSCS) under project ID s516. This work used the DiRAC Data Analytic system at the University of Cambridge, operated by the University of Cambridge High Performance Computing Service on behalf of the STFC DiRAC HPC Facility (www.dirac.ac.uk). This equipment was funded by BIS National E-infrastructure capital grant (ST/K001590/1), STFC capital grants ST/H008861/1 and ST/H00887X/1, and STFC DiRAC Operations grant ST/K00333X/1. DiRAC is part of the National EInfrastructure.

\section{References}

Bastian, T. S., Dulk, G. A., \& Leblanc, Y. 2000, ApJ, 545, 1058

Donati, J., Howarth, I. D., Jardine, M. M., et al. 2006, MNRAS, 370, 629

Donati, J.-F. \& Brown, S. F. 1997, A\& A, 326, 1135

Fares, R., Donati, J.-F., Moutou, C., et al. 2012, MNRAS, 423, 1006

Fares, R., Moutou, C., Donati, J.-F., et al. 2013, MNRAS, 435, 1451

Farrell, W. M., Desch, M. D., \& Zarka, P. 1999, JGR, 104, 14025

Grießmeier, J.-M., Motschmann, U., Mann, G., \& Rucker, H. O. 2005, A\&\&A, 437, 717

Grießmeier, J.-M., Zarka, P., \& Girard, J. N. 2011, Radio Science, 46, 0

Hallinan, G., Sirothia, S. K., Antonova, A., et al. 2013, ApJ, 762, 34

Jardine, M. \& Cameron, A. C. 2008, A\&SA, 490, 843

Lazio, T. J. W., Farrell, W. M., Dietrick, J., et al. 2004, ApJ, 612, 511

Lecavelier des Etangs, A., Sirothia, S. K., \& Gopal-Krishna, Zarka, P. 2013, A\& A, 552, A65

Llama, J., Vidotto, A. A., Jardine, M., et al. 2013, MNRAS, 436, 2179

Loesch, C., Opher, M., Alves, M. V., Evans, R. M., \& Manchester, W. B. 2011, JGR (Space Physics), 116, A04106

Parker, E. N. 1958, ApJ, 128, 664

Powell, K. G., Roe, P. L., Linde, T. J., Gombosi, T. I., \& de Zeeuw, D. L. 1999, J. of Comp. Physics, 154, 284

See, V., Jardine, M., Fares, R., Donati, J.-F., \& Moutou, C. 2015, MNRAS, 450, 4323

See, V., Jardine, M., Vidotto, A. A., et al. 2014, A\&\&A, 570, A99 
Sirothia, S. K., Lecavelier des Etangs, A., Gopal-Krishna, Kantharia, N. G., \& Ishwar-Chandra, C. H. 2014, $A \mathscr{E} A, 562, \mathrm{~A} 108$

Smith, A. M. S., Collier Cameron, A., Greaves, J., et al. 2009, MNRAS, 395, 335

Tóth, G., van der Holst, B., Sokolov, I. V., et al. 2012, Journal of Computational Physics, 231, 870

Van Doorsselaere, T., Wardle, N., Del Zanna, G., et al. 2011, ApJL, 727, L32

Vidotto, A. A., Fares, R., Jardine, M., et al. 2012, MNRAS, 423, 3285

Vidotto, A. A., Fares, R., Jardine, M., Moutou, C., \& Donati, J.-F. 2015, MNRAS, 449, 4117

Vidotto, A. A., Gregory, S. G., Jardine, M., et al. 2014a, MNRAS, 441, 2361

Vidotto, A. A., Jardine, M., \& Helling, C. 2011a, MNRAS, 411, L46

Vidotto, A. A., Jardine, M., Morin, J., et al. 2014b, MNRAS, 438, 1162

Vidotto, A. A., Llama, J., Jardine, M., Helling, C., \& Wood, K. 2011b, Astronomische Nachrichten, 332, 1055

Vidotto, A. A., Opher, M., Jatenco-Pereira, V., \& Gombosi, T. I. 2010, ApJ, 720, 1262

Zarka, P. 2007, Planetary Space Science, 55, 598 